\title{
Utility of scoring systems combining the product of tumor number and size with liver function for predicting the prognosis of patients with hepatocellular carcinoma after hepatectomy
}

\author{
YUKIO TOKUMITSU $^{1}$, YOSHITARO SHINDO ${ }^{1}$, HIROTO MATSUI $^{1}$, SATOSHI MATSUKUMA $^{1}$, \\ MASAO NAKAJIMA $^{1}$, NOBUAKI SUZUKI ${ }^{1}$, SHIGERU TAKEDA ${ }^{1}$, HIROSHI WADA ${ }^{2}$, \\ SHOGO KOBAYASHI ${ }^{3}$, HIDETOSHI EGUCHI ${ }^{3}$, TOMIO UENO ${ }^{4}$ and HIROAKI NAGANO ${ }^{1}$
}

\footnotetext{
${ }^{1}$ Department of Gastroenterological, Breast and Endocrine Surgery, Yamaguchi University Graduate School of Medicine, Ube, Yamaguchi 755-8505; ${ }^{2}$ Department of Digestive Surgery, Osaka International Cancer Institute, Osaka 541-8567;

${ }^{3}$ Department of Gastroenterological Surgery, Osaka University Graduate School of Medicine, Osaka 565-0871;

${ }^{4}$ Department of Digestive Surgery, Kawasaki Medical School, Kurashiki, Okayama 701-0192, Japan
}

Received January 13, 2019; Accepted July 2, 2019

DOI: $10.3892 / 01.2019 .10688$

\begin{abstract}
Our previous study reported the effectiveness of the product of tumor number and size $(\mathrm{NxS}$ factor) as a predictor of the prognosis of patients with hepatocellular carcinoma (HCC) following hepatectomy. The aim of the present study was to validate the prognostic value of scoring systems based on the NxS factor for HCC. The records of 940 patients who underwent hepatectomy for HCC at Osaka University Graduate School of Medicine and Osaka International Cancer Institute were analyzed. The discriminatory abilities of the mathematical integrated model for tumor staging (MITS) score, which combines the $\mathrm{NxS}$ factor with liver function, and known prognostic systems, including the Japan Integrated Staging system, the Barcelona Clinic Liver Cancer, Cancer of the Liver Italian Program and the Tokyo system, were compared. Firstly, the present study demonstrated that a higher NxS factor was associated with decreased disease-free survival (DFS) and overall survival (OS) in patients with HCC $(\mathrm{P}<0.05)$. Subsequently, univariate analysis of DFS and OS curves revealed significant differences among all NxS factor and liver damage combinations (MITS-LD score; score 0 vs. score 1 ; score 1 vs. score 2 ; score 2 vs. score 3 ; all $\mathrm{P}<0.05$ ) and all $\mathrm{NxS}$ factor and albumin-bilirubin (ALBI) score combinations (MITS-ALBI score; score 0 vs. score 1; score 1 vs. score 2; score 2 vs. score 3; all $\mathrm{P}<0.05$ ). Furthermore, multivariate Cox proportional hazards model analysis demonstrated
\end{abstract}

Correspondence to: Dr Yukio Tokumitsu, Department of Gastroenterological, Breast and Endocrine Surgery, Yamaguchi University Graduate School of Medicine, 1-1-1 Minamikogushi, Ube, Yamaguchi 755-8505, Japan

E-mail: yt790604@yamaguchi-u.ac.jp

Key words: hepatocellular carcinoma, staging system, prognostic factor, tumor number, tumor size that there were significant differences in DFS [Hazard Ratio (HR); score 0 vs. score 1 (1.48); score 1 vs. score 2 (1.27); score 2 vs. score 3 (1.64); all $\mathrm{P}<0.05$ ] and OS [HR; score 0 vs. score 1 (1.34); score 1 vs. score 2 (1.29); score 2 vs. score 3 (1.64); all $\mathrm{P}<0.05$ ] among patients with different MITS-LD scores, and there were significant differences-in DFS [HR; score 0 vs. score 1 (1.38); score 1 vs. score 2 (1.43); score 2 vs. score 3 (1.60); all $\mathrm{P}<0.05$ ] among patients with different MITS-ALBI scores. The NxS factor may be a comprehensive measure of tumor burden for predicting the prognosis of patients with HCC following liver resection, and MITS scores could be an improved scoring system for predicting the prognosis of patients with HCC after hepatectomy.

\section{Introduction}

Hepatocellular carcinoma (HCC) is one of the most common malignancies worldwide $(1,2)$. Although liver resection for HCC is now considered a safe approach $(3,4)$ and has the highest local controllability of all local treatments, the recurrence and death rates of HCC remain high even after curative hepatectomy Additionally, predicting the prognosis of HCC patients is more complex than that of patients with most solid tumors, because it depends on the tumor burden, in addition to the patient's underlying liver disease and liver functional reserve (5). Therefore, staging systems based on both tumor factors and host factors such as liver function have been required to accurately classify HCC patients undergoing various therapeutic options (6-8). Several staging systems/prognostic scores such as the Japan Integrated Staging score (JIS score) (9), modified JIS (M-JIS) score (10), Albumin-Bilirubin grade with TNM score (ALBI-T score) (11), the Cancer of the Liver Italian Program (CLIP) score (12), the Tokyo Score (13), and Barcelona Clinic liver cancer (BCLC) staging (14) have been proposed during the last two decades.

One of the goals of staging systems today is to provide an evidence-based treatment guide (14). Although all staging classifications have been designed to predict prognosis, many 
staging systems lack optimal treatment allocation except for BCLC. The BCLC classification is currently the most used in Western countries as a standard, and the precise description of treatment strategy is indicated at this stage. However, BCLC treatment recommendations were not suitable in all situations. In Japan, it is considered that the therapeutic algorithm in the Japanese guidelines for the management of liver cancer is established and superior to the BCLC treatment algorithm in Japanese population (15). Recently, the Hong Kong Liver Cancer (HKLC) classification was constructed by a Hong Kong group to developed treatment guidance for Asian patients with HCC (16). Among these countries, treatment situations and options are various in some part, thus, it seems to be currently difficult to establish the unified staging system which provides both optimal treatment recommendation and prediction prognosis for worldwide.

Another goal of staging systems is to develop a globally applicable staging classification (17). There is currently no globally accepted system for assessing HCC patients, due to the heterogeneity of the tumor extension and of underlying liver disease, and thus no common language on which to base treatment decisions and guide research. For practical purposes, staging systems should be simple and based on data that are easily obtainable.

We previously reported the effectiveness of the product of tumor number and size ( $\mathrm{NxS}$ factor), and we constructed a mathematical integrated model for tumor staging (MITS) score by combining the NxS factor with liver function to predict the prognosis of HCC patients who underwent curative hepatectomy at Yamaguchi University School of Medicine $(18,19)$. The aim of the current study is to perform external validation of scoring based on the NxS factor, which were very simple and may potentially become one of a common score in many countries, in terms of stratification ability compared with known prognostic systems.

\section{Materials and methods}

From 1990 to 2010, a total of 1063 HCC patients who underwent hepatectomy with a minimum follow-up of 5 years after surgery in Osaka University Hospital $(n=343)$ and Osaka International Cancer Center $(n=720)$ were eligible for this study. The indications for liver resection were in accordance with the Japanese guideline on liver cancer examination and treatment (20), which recommends hepatectomy for $\mathrm{HCC}$ with $\leq 3$ lesions if liver function is Liver damage A or $\mathrm{B}$, regardless of the tumor size in preoperative imaging modality.

The diagnoses of HCC were all confirmed pathologically. Curative hepatectomy was defined as complete resection of all tumor nodules without involving any major branch of the portal or hepatic veins. Patients were excluded from the analysis if they met any of the following criteria: Volume reduction surgery or $\mathrm{HCC}$ with thrombosis of a major branch of the portal or hepatic vein $(n=35)$, distant metastasis $(n=1)$, Child-Pugh classification $\mathrm{C}$ and/or liver damage classification $C(n=5)$, death within 90 days after surgery $(n=3)$, death from other diseases $(n=8)$, information about recurrence not collected $(n=6)$, and insufficient clinical data $(n=65)$. Finally, 940 patients were enrolled in the study. All patients were followed-up after hepatectomy until death or the date of the last follow-up visit, and survival was censored in December 2015.

Data on tumor factors, such as the maximum size of the main tumor, number of tumors, differentiation, and microvascular invasion, were based on the final pathological findings of the resected liver. Laboratory data, including serum bilirubin, albumin, prothrombin activity, platelet count, indocyanine green retention rate at 15 min (ICG-R15), hepatitis B surface antigen, anti-hepatitis $\mathrm{C}$ antibody, and alpha-fetoprotein (AFP), were obtained before operation. The Child-Pugh classification (21), the degree of liver damage classification by the Liver Cancer Study Group of Japan (LCSGJ) (22), the ALBI grade (23), JIS score, M-JIS score, ALBI-T score, CLIP score, Tokyo score, and BCLC staging system were evaluated using these variables.

The NxS factor, as a marker of tumor burden of the MITS score, was incorporated with the parameters used to assess liver functions such as the Child-Pugh classification (MITS-CP score), the degree of liver damage classification (MITS-LD score), and the ALBI grade (MITS-ALBI score) (Tables I-IV). The NxS factor was categorized into three groups $(<4,4-9$, and $>9$ ). We had determined the optimal cut-off values of the NxS factor at 4 and 9 in reference to the Milan criteria (single tumor $\leq 5 \mathrm{~cm}$ in size or $\leq 3$ tumors each $\leq 3 \mathrm{~cm}$ in size) (24), and in the previous report, we had demonstrated that cut off value of NxS factor at 4 and 9 had high accuracy in predicting in recurrence of $\mathrm{HCC}$, and given that the $\mathrm{NxS}$ factor and the degree of Liver Damage classification by LCSGJ were independent risk factors for HCC prognosis by multivariate analysis (19).

In the MITS score, single-digit values were assigned to each group to show the disparity in severity. Patients with $\mathrm{NxS}$ factors $<4,4-9$, and $>9$ were assigned the single-digit values of 0,1 , and 2 , respectively. Patients with Child-Pugh and the degree of liver damage classes $\mathrm{A}, \mathrm{B}$, and $\mathrm{C}$ were assigned the single-digit values of 0,1 , and 2 , respectively. Similarly, patients with ALBI grades 1, 2, and 3 were assigned the single-digit values of 0,1 and 2 , respectively.

The MITS-CP score was obtained via the summation of the NxS value and the Child-Pugh value, the MITS-LD score was obtained via the summation of the $\mathrm{NxS}$ value and the liver damage value, and the MITS-ALBI score was obtained via the summation of the NxS value and ALBI value, respectively.

This retrospective study was approved by Institutional Review Board of Yamaguchi University Hospital [H27-141(December 22, 2015)], and the retrospectively collected data were analyzed statistically and assessed at Yamaguchi University.

Statistical analysis. The variables analyzed were patient age, sex, liver function, tumor factors and scores of the systems. Continuous variables were expressed as the median \pm standard error. Disease-free survival (DFS) and overall survival (OS) curves were plotted with the Kaplan-Meier method, and differences in DFS and OS between the groups were compared using Generalized Wilcoxon Test on univariate analysis. The Holm correction for multiple comparisons was applied. Staging systems for HCC were tested by multivariate Cox analyses of both DFS and OS. P-values less than 0.05 were considered statistically significant. Statistical analysis 
Table I. NxS factor.

\begin{tabular}{lccc}
\hline Model & Formula & Calculated value (points) & Classification (score) \\
\hline NxS factor & Tumor number $(\mathrm{n}) \mathrm{x}$ tumor size $(\mathrm{cm})$ & $<4$ & 0 \\
& & $4-9$ & 1 \\
& & $>9$ & 2 \\
\hline
\end{tabular}

$\mathrm{NxS}$, product of tumor number and size.

Table II. ALBI grade.

\begin{tabular}{lccc}
\hline Model & Formula & Calculated value (points) & Classification (grade) \\
\hline ALBI grade & $\log _{10}$ T-bil $(\mathrm{mM}) \times 0.66+\mathrm{ALB}(\mathrm{g} / \mathrm{l}) \mathrm{x}-0.0852$ & $\leq-2.16$ & 1 \\
& & $>-2.16,<-1.39$ & 2 \\
& & $>-1.39$ & 3 \\
\hline
\end{tabular}

ALB, albumin; ALBI, albumin-bilirubin; T-bil, total bilirubin.

Table III. Liver damage classification by LCSGJ .

\begin{tabular}{lccc}
\hline & \multicolumn{3}{c}{$\begin{array}{c}\text { Liver damage classification } \\
\text { according to LCSGJ }\end{array}$} \\
\cline { 2 - 4 } Item & $\mathrm{A}$ & $\mathrm{B}$ & $\mathrm{C}$ \\
\hline Ascites & None & Controllable & Uncontrollable \\
Bilirubin (mg/dl) & $<2.0$ & $2.0-3.0$ & $>3.0$ \\
Albumin $(\mathrm{g} / \mathrm{dl})$ & $>3.5$ & $3.0-3.5$ & $<3.0$ \\
ICG R15 (\%) & $<15$ & $15-40$ & $>40$ \\
Prothrombin activity $(\%)$ & $>80$ & $50-80$ & $<50$
\end{tabular}

The highest grade for which at least two items meet the criteria is adopted as the degree of liver damage. ICG R15, indocyanine green retention rate at $15 \mathrm{~min}$; LCSGJ, Liver Cancer Study Group of Japan.

was performed using JMP version 13.0 (SAS Institute Japan, Tokyo, Japan).

\section{Results}

The baseline characteristics of the $940 \mathrm{HCC}$ patients are shown in Table V. Of the 940 patients, 668 developed HCC recurrence within 5 years after hepatectomy. The disease-free median survival time was 26.8 months [95\% confidence interval (CI), 24.8 to 30.1 months], and the 1-, 3-, and 5 -year DFS rates were $73.0,42.1$, and $28.4 \%$, respectively. Furthermore, 320 patients died within 5 years after hepatectomy. The OS time was 82.8 months $(95 \% \mathrm{CI}, 76.5$ to 88.8 months), and the 1-, 3-, and 5-year OS rates were 95.6, 80.0 , and $66.0 \%$, respectively.

The 1-year DFS rates were $80.8,68.6$, and $47.2 \%$, the 3 -year DFS rates were 50.5, 35.4, and $21.1 \%$, and the 5-year DFS rates were $33.3,24.6$, and $15.4 \%$ in patients with $\mathrm{NxS}$ factor $<4$,
Table IV. Three MITS scores that combine the NxS factor and liver function.

\begin{tabular}{lccc}
\hline & \multicolumn{3}{c}{ Value } \\
\cline { 2 - 4 } Scoring system & 0 & 1 & 2 \\
\hline MITS-CP score & & & \\
NxS factor & 4 & $4-9$ & $>9$ \\
Child-Pugh classification & $\mathrm{A}$ & $\mathrm{B}$ & $\mathrm{C}$ \\
MITS-LD score & & & \\
$\quad$ NXS factor & $<4$ & $4-9$ & $>9$ \\
Liver damage classification & $\mathrm{A}$ & $\mathrm{B}$ & $\mathrm{C}$ \\
MITS-ALBI score & & & \\
$\quad$ NxS factor & $<4$ & $4-9$ & $>9$ \\
ALBI grade & 1 & 2 & 3 \\
\hline
\end{tabular}

The MITS-CP score was obtained via the summation of the NxS and the Child-Pugh values, the MITS-LD score was obtained via the summation of the $\mathrm{NxS}$ and the liver damage values, and the MITS-ALBI score was obtained via the summation of the $\mathrm{NxS}$ and ALBI values. ALBI, albumin-bilirubin; MITS-CP, mathematical integrated model for tumor staging with the Child-Pugh classification; MITS-LD, mathematical integrated model for tumor staging with the degree of liver damage classification; MITS-ALBI, mathematical integrated model for tumor staging with the albumin-bilirubin grade; NxS, product of tumor number and size.

$\mathrm{NxS}$ factor 4-9, and $\mathrm{NxS}$ factor $>9$, respectively. The 1-year OS rates were 98.1, 95.2, and 85.2\%, the 3-year OS rates were 85.9, 79.0, and 54.6\%, and the 5-year OS rates were 73.8, 59.9, and $45.4 \%$ in patients with $\mathrm{NxS}$ factor $<4, \mathrm{NxS}$ factor $4-9$, and $\mathrm{NxS}$ factor $>9$, respectively. There were significant differences in DFS and OS among the groups classified by NxS factor (all $\mathrm{P}<0.05)$. 
Table V. Patient profiles and tumor characteristics.

\begin{tabular}{|c|c|c|}
\hline Variable & Number of patients, $n,(n=940)$ & Percentage of patients, $\%$ \\
\hline \multicolumn{3}{|l|}{ Host factor } \\
\hline Age, years (mean \pm standard error) & $64.2 \pm 0.28$ & \\
\hline Sex (male/female) & $723 / 217$ & $(76.9 / 23.1)$ \\
\hline HBs Ag (positive/negative/unknown) & $168 / 755 / 17$ & $(17.9 / 80.3 / 1.8)$ \\
\hline HCV Ab (positive/negative/unknown) & $627 / 305 / 8$ & $(66.7 / 32.4 / 0.9)$ \\
\hline Bilirubin, mg/dl (mean \pm standard error) & $0.81 \pm 0.01$ & \\
\hline Albumin, g/dl (mean \pm standard error) & $3.86 \pm 0.04$ & \\
\hline Prothrombin time, $\%$ (mean \pm standard error) & $84.3 \pm 0.42$ & \\
\hline ICG-R15, \% (mean \pm standard error) & $17.9 \pm 0.35$ & \\
\hline Platelet count, $\mathrm{x} 10^{3} / \mathrm{mm}^{3}$ (mean \pm standard error) & $15.3 \pm 0.26$ & \\
\hline Degree of liver damage (A/B) & $651 / 289$ & $(69.3 / 30.7)$ \\
\hline Child-Pugh classification (A/B) & $865 / 75$ & $(92.0 / 8.0)$ \\
\hline ALBI grade (A/B) & $548 / 392$ & $(58.3 / 41.7)$ \\
\hline \multicolumn{3}{|l|}{ Tumor factor } \\
\hline Tumor maximum diameter, $\mathrm{cm}(<2 />2,<5 / \geq 5)$ & $182 / 586 / 172$ & $(19.4 / 62.3 / 18.3)$ \\
\hline Number of tumors $(1 / 2-3 / \geq 4)$ & $732 / 188 / 20$ & $(77.9 / 20.0 / 2.1)$ \\
\hline $\mathrm{NxS}$ factor $(<4 / 4-9 />9)$ & $523 / 309 / 108$ & $(55.6 / 32.9 / 11.5)$ \\
\hline Macroscopic portal vein invasion (absent/present/unknown) & $885 / 39 / 16$ & $(94.1 / 4.1 / 1.7)$ \\
\hline Macroscopic hepatic vein invasion (absent/present/unknown) & $931 / 6 / 3$ & $(99.0 / 0.6 / 0.3)$ \\
\hline Microscopic portal vein invasion (absent/present) & $302 / 638$ & $(32.1 / 67.9)$ \\
\hline Microscopic hepatic vein invasion (absent/present) & $899 / 41$ & $(95.6 / 4.4)$ \\
\hline AFP, ng/ml $(<400 / \geq 400)$ & $761 / 179$ & $(81.0 / 19.0)$ \\
\hline DCP, mAU/ml (<1,000/>1,000/unknown) & $713 / 164 / 63$ & $(75.9 / 174.5 / 6.7)$ \\
\hline Differentiation (well/moderate/poor/unknown) & $134 / 575 / 211 / 20$ & $(14.3 / 61.2 / 22.4 / 2.1)$ \\
\hline Anatomical resection (yes/no) & $466 / 474$ & $(49.6 / 50.4)$ \\
\hline Recurrence (yes/no) & $714 / 226$ & $(76.0 / 24.0)$ \\
\hline Death (yes/no) & $576 / 364$ & $(61.3 / 38.7)$ \\
\hline TNM Stage, LCSGJ 5th (I/II/III/IV) & $165 / 383 / 325 / 67$ & $(17.6 / 40.7 / 34.6 / 7.1)$ \\
\hline TNM Stage, AJCC 8th (IA/IB/II/III/IVA) & $197 / 325 / 360 / 56 / 2$ & $(21.0 / 34.6 / 38.3 / 6.0 / 0.2)$ \\
\hline \multicolumn{3}{|l|}{ Staging systems } \\
\hline MITS-CP model $(0 / 1 / 2 / 3)$ & $483 / 324 / 123 / 10$ & $(51.4 / 34.5 / 13.1 / 1.1)$ \\
\hline MITS-LD model $(0 / 1 / 2 / 3)$ & $367 / 366 / 173 / 34$ & $(39.0 / 39.0 / 18.4 / 3.6)$ \\
\hline MITS-ALBI model (0/1/2/3) & $214 / 437 / 231 / 58$ & $(22.8 / 46.5 / 24.6 / 6.2)$ \\
\hline JIS score $(0 / 1 / 2 / 3 / 4)$ & $151 / 364 / 337 / 81 / 7$ & $(16.1 / 38.7 / 35.9 / 8.6 / 0.7)$ \\
\hline Modified JIS score $(0 / 1 / 2 / 3 / 4)$ & $116 / 316 / 340 / 145 / 23$ & $(12.3 / 33.6 / 36.2 / 15.4 / 2.4)$ \\
\hline ALBI-T score $(0 / 1 / 2 / 3 / 4)$ & $67 / 261 / 351 / 225 / 36$ & $(7.1 / 27.8 / 37.3 / 23.9 / 3.8)$ \\
\hline Tokyo score $(0 / 1 / 2 / 3 / 4)$ & $97 / 376 / 342 / 112 / 13$ & $(10.3 / 40.0 / 36.4 / 11.9 / 1.4)$ \\
\hline $\operatorname{CLIP}(0 / 1 / 2 / 3 / 4 / 5)$ & $439 / 323 / 119 / 43 / 11 / 5$ & $(46.7 / 34.4 / 12.7 / 4.6 / 0.5)$ \\
\hline $\mathrm{BCLC}(0 / \mathrm{A} / \mathrm{B} / \mathrm{C})$ & $144 / 661 / 96 / 39$ & $(15.3 / 70.3 / 10.2 / 4.1)$ \\
\hline
\end{tabular}

AFP, $\alpha$-fetoprotein; ALBI-T, Albumin-Bilirubin grade with TNM; BCLC, Barcelona Clinic Liver Cancer; CLIP, Cancer of the Liver Italian Program; HBs Ag, hepatitis B surface antigen; HCV Ab, hepatitis C virus antibody; ICG-R15, indocyanine green retention rate at 15 min; JIS, Japan Integrated Staging score; LCSGJ, Liver Cancer Study Group of Japan; MITS-CP, mathematical integrated model for tumor staging with the Child-Pugh classification; MITS-LD, mathematical integrated model for tumor staging with the degree of liver damage classification, MITS-ALBI, mathematical integrated model for tumor staging with Albumin-Bilirubin grade; NxS, tumor number and size; TNM, tumor-lymph node-metastasis; UICC, Union for International Cancer Control.

The distribution of the NxS factor for each $\mathrm{T}$ factor of the TNM staging systems (LCSGJ and UICC) is shown in Fig. 1. The patients classified as T1 of TNM (LCSGJ) and T1a of TNM (UICC) consisted of 166 and 198 patients, respectively; all had NxS factor <4. Moreover, most patients with T1/T2 of TNM (LCSGJ) and T1 (T1a/T1b) of TNM (UICC) had $\mathrm{NxS}$ factor $<4$. On the other hand, the patients classified as T3 of TNM (UICC) consisted of 56 patients, all of who had 

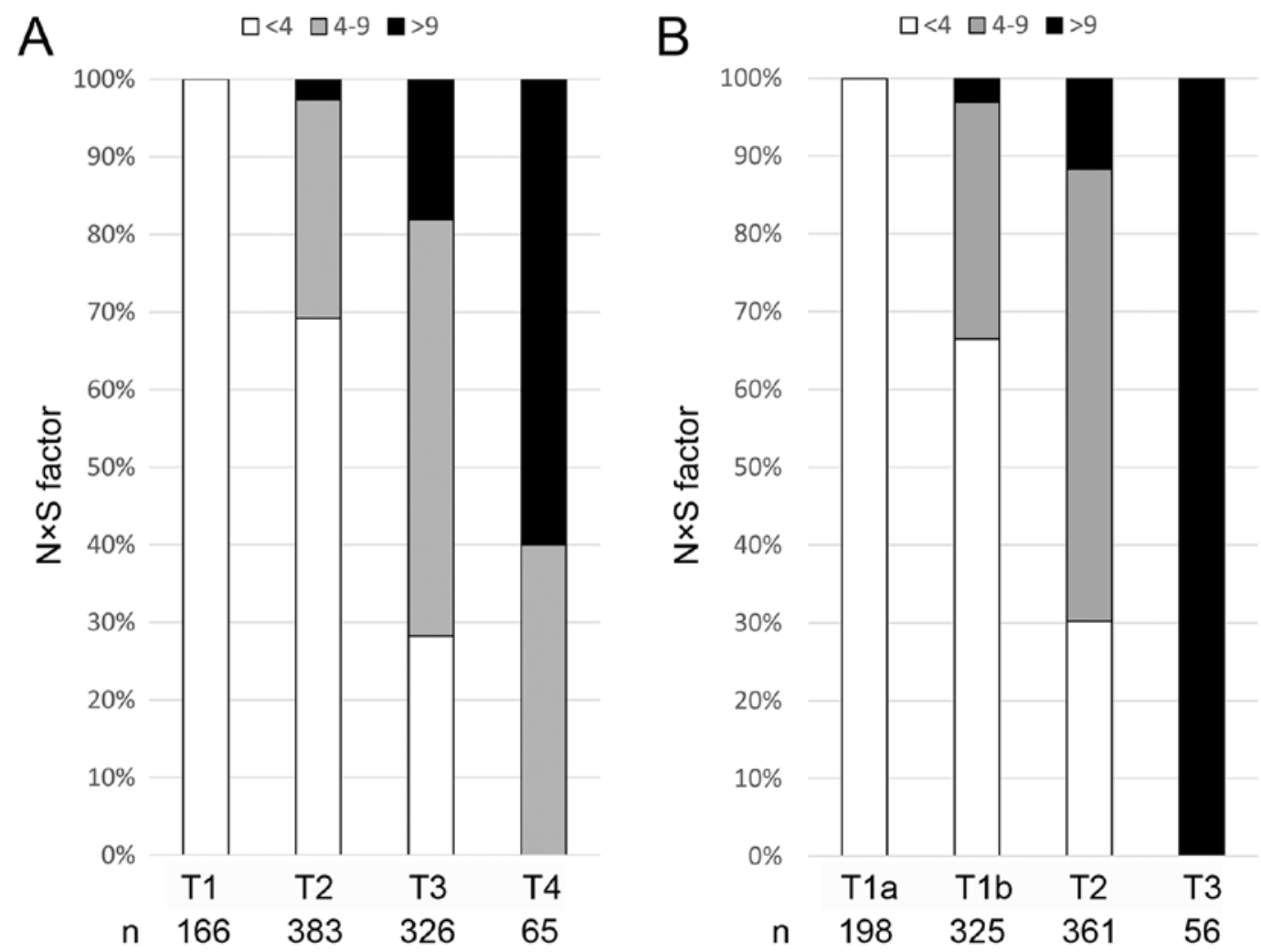

Figure 1. Distribution of the NxS factor for the T factor. (A) Distribution of the NxS factor for the T factor of the TNM staging systems (LCSGJ). The group of patients classified as T1 consisted of 166 patients, all of whom exhibited an NxS factor <4. In addition, the majority of patients with T1/T2 had an NxS factor <4. (B) Distribution of the NxS factor for the T factor of the TNM staging systems (UICC). The group of patients classified as T1a consisted of 198 patients, all of whom had an NxS factor <4. In addition, the majority of patients with T1 (T1a/T1b) had an NxS factor <4. However, the group of patients classified as $\mathrm{T} 3$ consisted of 56 patients, all of whom had an $\mathrm{NxS}>9$. The distribution of the NxS factor among patients with T1, T2 and T3 of the TNM staging system (LCSGJ) was similar to that among patients with T1a, T1b and T2 of the TNM staging system (UICC). LCSGJ, Liver Cancer Study Group of Japan; NxS, product of tumor number and size; UICC, Union for International Cancer Control.

$\mathrm{NxS}>9$. The distribution of the NxS factor among patients with T1, T2, and T3 of the TNM staging system (LCSGJ) was similar to that of T1a, T1b, and T2 of the TNM staging system (UICC), respectively. On the whole, the distribution of the $\mathrm{NxS}$ factor was relevant to that of T factor of the TNM staging systems, especially UICC 8th.

In the present study, no patients were classified as Child-Pugh C, Liver Damage C, and ALBI grade 3. Although there were not significant differences in DFS between the Child-Pugh A and B, there were significant differences in OS between the Child-Pugh A and B $(\mathrm{P}<0.05)$. Meanwhile, there were significant differences in DFS and OS between the degree of liver damage $\mathrm{A}$ and $\mathrm{B}$, and there were also significant differences in DFS and OS between the ALBI grade A and $\mathrm{B}$.

The Kaplan-Meier estimated DFS curves and OS curves according to the three MITS scores, the JIS score, the M-JIS score, the ALBI-T score, the Tokyo score, the CLIP score, and the BCLS staging system are shown in Figs. 2 and 3, respectively. In univariate analysis, there were significant differences (all $\mathrm{P}<0.05)$ in both DFS and OS between patients with different MITS-LD scores (score 0 vs. score 1; score 1 vs. score 2; score 2 vs. score 3 ) and MITS-ALBI scores (score 0 vs. score 1 ; score 1 vs. score 2 ; score 2 vs. score 3 ), respectively. Of the known prognostic scores, the M-JIS score had good stratification abilities for both DFS and OS. There were significant differences in DFS (all $\mathrm{P}<0.05)$ between patients with different M-JIS scores (score 0 vs. score 1, score 1 vs. score 2; score 2 vs. score 3; score 3 vs. score 4 ), and there were significant differences (all $\mathrm{P}<0.05$ ) in $\mathrm{OS}$ between patients with different M-JIS scores (score 0 vs. score 1; score 1 vs. score 2 ; score 2 vs. score 3 ), but there was a marginal difference between patients with score 3 and those with score 4 $(\mathrm{P}=0.0644)$. Among the nine staging systems, the MITS-LD score and the MITS-ALBI score demonstrated significant differences in DFS and OS between all adjacent strata. The Table VI shows multivariate Cox proportional hazards regression analyses of the associations between staging systems and survival rates. There were also significant differences (all $\mathrm{P}<0.05)$ in both DFS and OS between patients with different MITS-LD scores (score 0 vs. score 1; score 1 vs. score 2; score 2 vs. score 3). Similarly, there were significant differences (all $\mathrm{P}<0.05$ ) in DFS between patients with different MITS-ALBI scores (score 0 vs. score 1; score 1 vs. score 2; score 2 vs. score 3 ). There were significant differences (all $\mathrm{P}<0.05)$ in OS between patients with different MITS-ALBI scores (score 1 vs. score 2; score 2 vs. score 3), but there was a marginally significant difference between patients with score 0 and those with score $1(\mathrm{P}=0.0942)$. Among the nine staging systems, only the MITS-LD score and demonstrated significant differences in DFS and OS between all adjacent strata. 
A
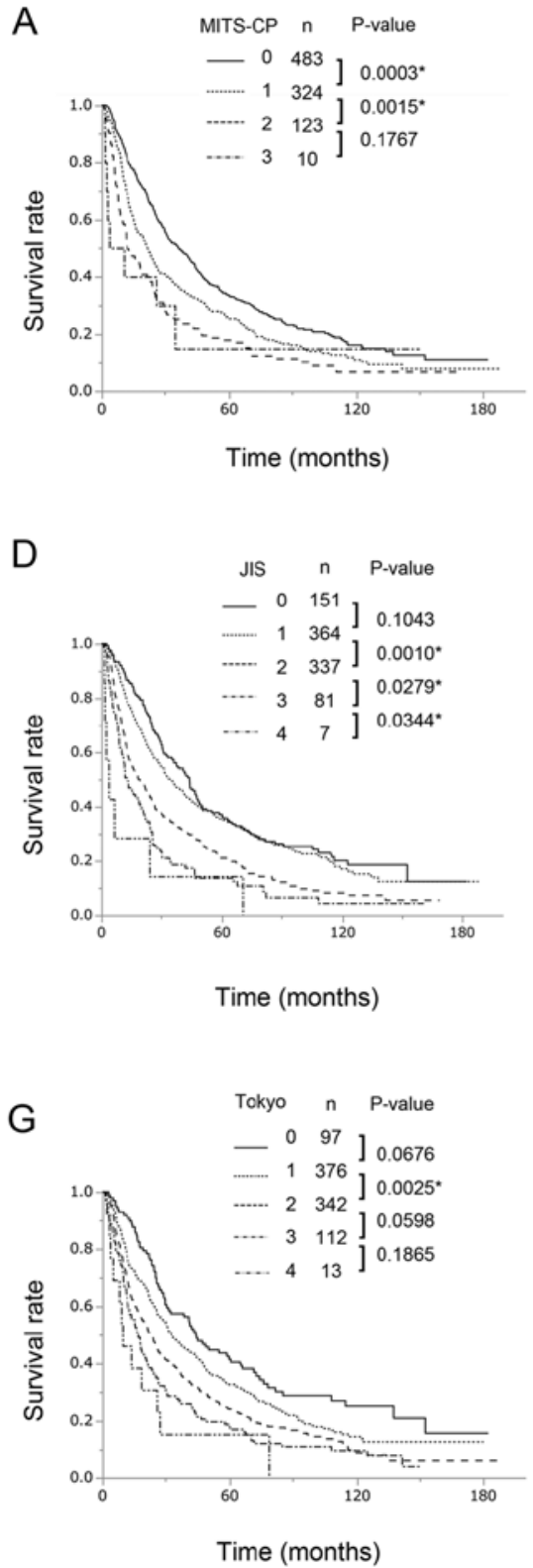

B

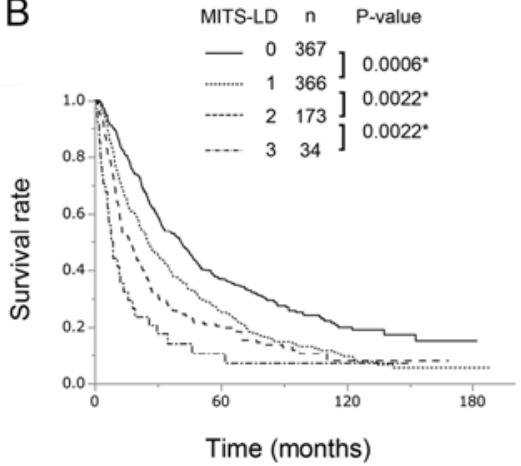

$\mathrm{E}$

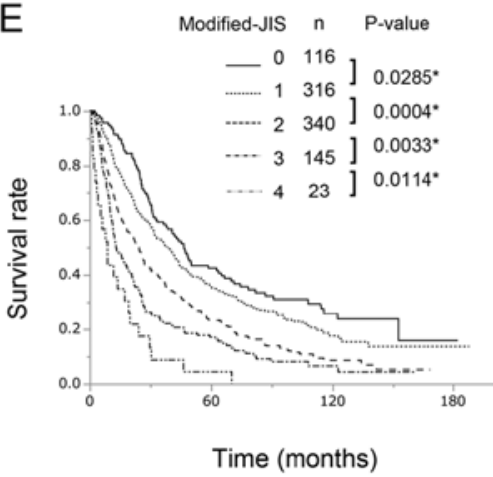

$\mathrm{H}$

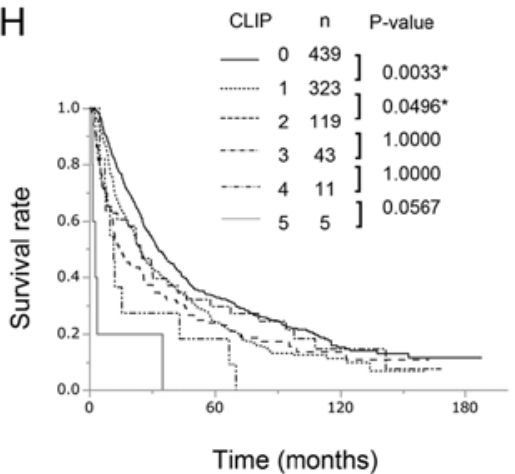

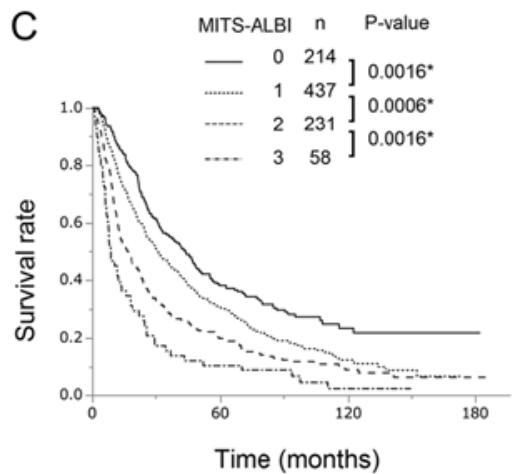

$\mathrm{F}$
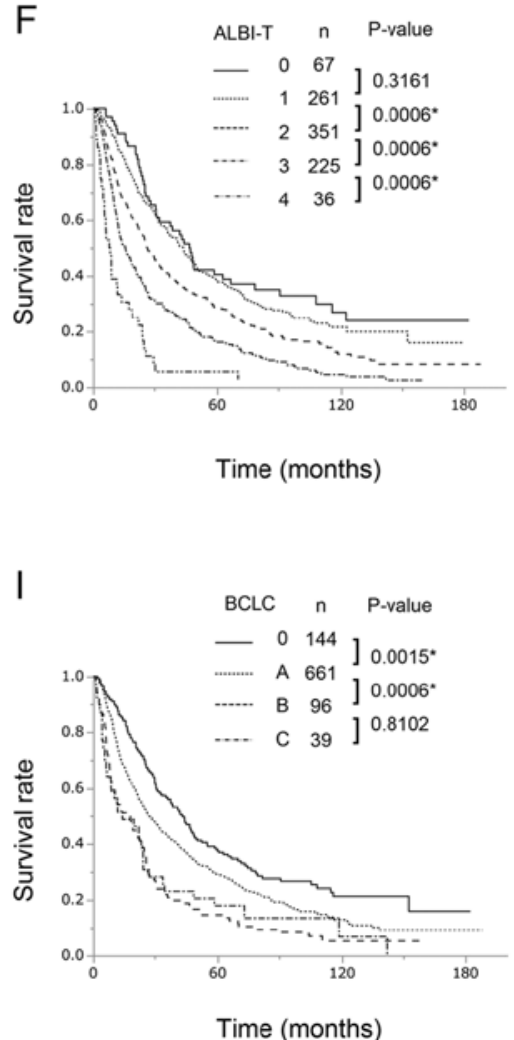

Figure 2. Comparison of DFS by scoring system. (A) MITS-CP score, (B) MITS-LD score, (C) MITS-ALBI score, (D) JIS score, (E) modified-JIS score, (F) ALBI-T score, (G) Tokyo score, (H) CLIP score and (I) BCLC staging system. The MITS-LD score and the MITS-ALBI score exhibited significant differences among all adjacent strata. ${ }^{*} \mathrm{P}<0.05$. DFS, disease-free survival. MITS-CP, mathematical integrated model for tumor staging with the Child-Pugh classification; MITS-LD, mathematical integrated model for tumor staging with the degree of liver damage classification; MITS-ALBI, mathematical integrated model for tumor staging with Albumin-Bilirubin grade; JIS, Japan Integrated Staging; ALBI-T, Albumin-Bilirubin grade with TNM; CLIP, Cancer of the Liver Italian Program; BCLC, Barcelona Clinic Liver Cancer.

\section{Discussion}

Staging systems based on information about both tumor factors and liver function are required to accurately classify HCC patients. External validation of the scores that combined the NxS factor and liver function was performed in terms of stratification ability in comparison with the known prognostic systems, and the most important finding of the present study was that the MITS-LD score had better stratification ability than that of other known prognostic systems for both DFS and OS. Although we had previously reported that the NxS of the largest tumor (i.e. the $\mathrm{NxS}$ factor) was a useful tumor factor to predict prognostic outcomes of HCC patients after curative hepatectomy $(19,25)$, and the score obtained by combining the
$\mathrm{NxS}$ factor with the degree of the liver damage classification provided good stratification abilities for DFS (19), there had been a need for external validation of the score. The current study demonstrated the utility of the scoring system that combined the NxS factor with the degree of the liver damage classification to predict both DFS and OS of HCC patients after hepatectomy.

The current study provided validation that the NxS factor, with optimal cut-off values determined to be 4 and 9 , was a useful tumor factor to predict HCC prognosis, and these cut-off points were determined in reference to the Milan criteria (single tumor $\leq 5 \mathrm{~cm}$ in size or $\leq 3$ tumors each $\leq 3 \mathrm{~cm}$ in size) (24) in the previous study (19), and classified the HCC patients into 3 groups showing different prognoses. All HCC patients with 

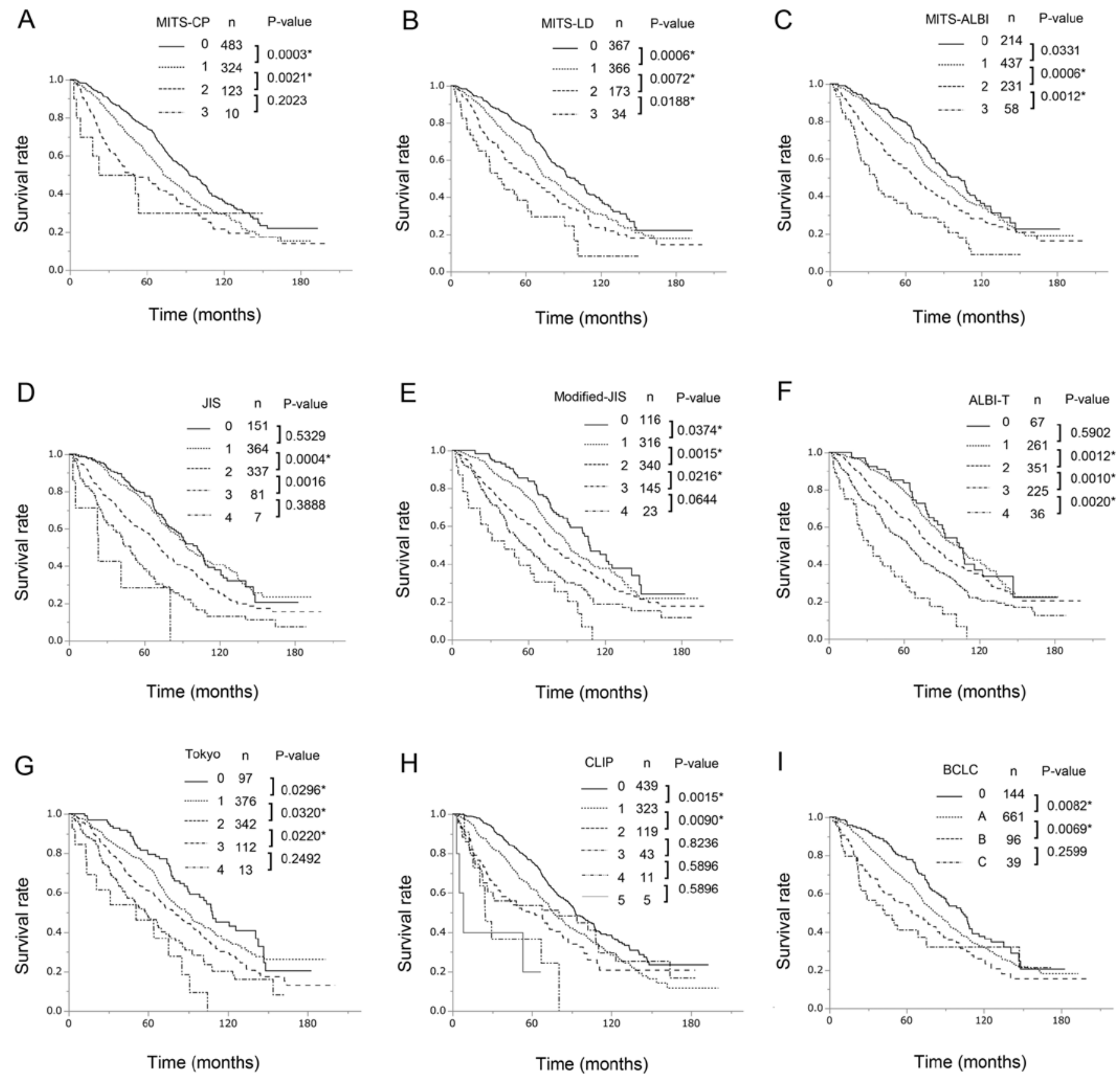

Figure 3. Comparison of OS by scoring system. (A) MITS-CP score, (B) MITS-LD score, (C) MITS-ALBI score, (D) JIS score, (E) M-JIS score, (F) ALBI-T score, (G) Tokyo score, (H) CLIP score and (I) BCLC staging system. The MITS-LD score exhibited significant differences among all adjacent strata. ${ }^{*} \mathrm{P}<0.05$. OS, overall survival. MITS-CP, mathematical integrated model for tumor staging with the Child-Pugh classification; MITS-LD, mathematical integrated model for tumor staging with the degree of liver damage classification; MITS-ALBI, mathematical integrated model for tumor staging with Albumin-Bilirubin grade; JIS, Japan Integrated Staging; M-JIS, modified Japan Integrated Staging; ALBI-T, Albumin-Bilirubin grade with TNM; CLIP, Cancer of the Liver Italian Program; BCLC, Barcelona Clinic Liver Cancer.

NxS factor $<4$ were within the Milan criteria and had a low incidence of recurrence, almost all patients with $\mathrm{NxS}$ factor $>9$ were outside the Milan criteria and had a high recurrence rate, and the remaining $\mathrm{HCC}$ patients with $\mathrm{NxS}$ factor 4-9 were considered to be at intermediate risk for $\mathrm{HCC}$ recurrence. Since the Milan criteria were first proposed in 1996, they became a cornerstone in the management of $\mathrm{HCC}$ patients and were partially or fully incorporated into several staging systems, such as TNM or BCLC. Until now, there has been an interest in changing how tumor morphological data are used to predict HCC prognosis. Mazaaferro proposed the 'Metroticket paradigm' (26) to express such a concept, that the longer the trip (i.e. an increase in tumor size and/or number of tumors) resulted in a higher ticket price (i.e. reduction in expected survival), and developed the Up-to-7 criteria (27) (ie, the sum of the largest tumor diameter in centimeters and the number of tumors is $<7$ ), which is one of the most popular liver transplantation criteria for patients who present with HCC beyond the Milan criteria. Total tumor volume (28), defined as the sum of the volume of each tumor [(4/3) x $3.14 \times$ (radius of the tumor in $\mathrm{cm})^{3}$ ], has also been an area of interest. Our NxS factor was similar in concept to the Metroticket paradigm, successfully 
Table VI. Multivariate Cox proportional hazards regression analyses of the associations between staging systems and survival rates.

\begin{tabular}{|c|c|c|c|c|c|c|}
\hline \multirow[b]{2}{*}{ Modeldel } & \multicolumn{3}{|c|}{ DFS } & \multicolumn{3}{|c|}{ OS } \\
\hline & HR & $95 \% \mathrm{CI}$ & P-value & HR & $95 \%$ CI & P-value \\
\hline \multicolumn{7}{|l|}{ MITS-CP } \\
\hline 0 vs. 1 & 1.36 & $1.16-1.58$ & $0.0001^{\mathrm{a}}$ & 1.37 & $1.15-1.64$ & $0.0006^{\mathrm{a}}$ \\
\hline 1 vs. 2 & 1.38 & $1.10-1.71$ & $0.0056^{\mathrm{a}}$ & 1.34 & $1.04-1.72$ & $0.0232^{\mathrm{a}}$ \\
\hline 2 vs. 3 & 1.05 & $0.47-2.02$ & 0.8978 & 1.30 & $0.54-2.60$ & 0.5253 \\
\hline \multicolumn{7}{|l|}{ MITS-LD } \\
\hline 0 vs. 1 & 1.48 & $1.26-1.74$ & $<0.0001^{\mathrm{a}}$ & 1.34 & $1.11-1.62$ & $0.0025^{\mathrm{a}}$ \\
\hline 1 vs. 2 & 1.27 & $1.04-1.54$ & $0.0187^{\mathrm{a}}$ & 1.29 & $1.03-1.61$ & $0.0257^{\mathrm{a}}$ \\
\hline 2 vs. 3 & 1.64 & $1.10-2.38$ & $0.0174^{\mathrm{a}}$ & 1.64 & $1.06-2.45$ & $0.0281^{\mathrm{a}}$ \\
\hline \multicolumn{7}{|c|}{ MITS-ALBI } \\
\hline 0 vs. 1 & 1.38 & $1.14-1.67$ & $0.0007^{\mathrm{a}}$ & 1.21 & $0.97-1.51$ & 0.0942 \\
\hline 1 vs. 2 & 1.43 & $1.21-1.70$ & $<0.0001^{\mathrm{a}}$ & 1.35 & $1.11-1.64$ & $0.0031^{\mathrm{a}}$ \\
\hline 2 vs. 3 & 1.60 & $1.18-2.13$ & $0.0030^{\mathrm{a}}$ & 1.80 & $1.29-2.47$ & $0.0008^{a}$ \\
\hline \multicolumn{7}{|l|}{ JIS } \\
\hline 0 vs. 1 & 1.11 & $0.90-1.38$ & 0.3301 & 1.01 & $0.78-1.30$ & 0.9453 \\
\hline 1 vs. 2 & 1.58 & $1.34-1.86$ & $<0.0001^{\mathrm{a}}$ & 1.54 & $1.27-1.87$ & $<0.0001^{\mathrm{a}}$ \\
\hline 2 vs. 3 & 1.38 & $1.07-1.78$ & $0.0152^{\mathrm{a}}$ & 1.60 & $1.22-2.10$ & $0.0012^{\mathrm{a}}$ \\
\hline 3 vs. 4 & 1.80 & $0.83-3.92$ & 0.1686 & 1.88 & $0.81-4.32$ & 0.1759 \\
\hline \multicolumn{7}{|c|}{ Modified-JIS } \\
\hline 0 vs. 1 & 1.26 & $0.98-1.62$ & 0.0676 & 1.29 & $0.96-1.74$ & 0.0809 \\
\hline 1 vs. 2 & 1.48 & $1.25-1.75$ & $<0.0001^{\mathrm{a}}$ & 1.31 & $1.07-1.60$ & $0.0078^{\mathrm{a}}$ \\
\hline 2 vs. 3 & 1.38 & $1.12-1.70$ & $0.0026^{\mathrm{a}}$ & 1.40 & $1.11-1.76$ & $0.0056^{\mathrm{a}}$ \\
\hline 3 vs. 4 & 1.92 & $1.23-2.99$ & $0.0079^{\mathrm{a}}$ & 1.70 & $1.06-2.72$ & $0.0367^{\mathrm{a}}$ \\
\hline \multicolumn{7}{|l|}{ ALBI-T } \\
\hline 0 vs. 1 & 1.17 & $0.84-1.61$ & 0.3440 & 1.03 & $0.71-1.50$ & 0.8629 \\
\hline 1 vs. 2 & 1.40 & $1.17-1.68$ & $0.0002^{\mathrm{a}}$ & 1.34 & $1.08-1.67$ & $0.0068^{\mathrm{a}}$ \\
\hline 2 vs. 3 & 1.52 & $1.27-1.81$ & $<0.0001^{\mathrm{a}}$ & 1.40 & $1.14-1.72$ & $0.0013^{\mathrm{a}}$ \\
\hline 3 vs. 4 & 2.10 & $1.47-3.02$ & $0.0002^{\mathrm{a}}$ & 2.19 & $1.49-3.20$ & $0.0002^{\mathrm{a}}$ \\
\hline \multicolumn{7}{|l|}{ Tokyo } \\
\hline 0 vs. 1 & 1.34 & $1.03-1.74$ & $0.0242^{\mathrm{a}}$ & 1.30 & $0.96-1.77$ & 0.0809 \\
\hline 1 vs. 2 & 1.29 & $1.10-1.52$ & $0.0018^{\mathrm{a}}$ & 1.28 & $1.06-1.54$ & $0.0111^{\mathrm{a}}$ \\
\hline 2 vs. 3 & 1.25 & $1.00-1.57$ & 0.0511 & 1.40 & $1.09-1.80$ & $0.0114^{\mathrm{a}}$ \\
\hline 3 vs. 4 & 1.58 & $0.87-2.87$ & 0.1607 & 1.66 & $0.90-3.03$ & 0.1253 \\
\hline \multicolumn{7}{|l|}{ CLIP } \\
\hline 0 vs. 1 & 1.31 & $1.12-1.54$ & $0.0008^{\mathrm{a}}$ & 1.38 & $1.15-1.66$ & $0.0007^{\circ}$ \\
\hline 1 vs. 2 & 1.14 & $0.91-1.44$ & 0.2588 & 1.32 & $1.02-1.70$ & $0.0395^{\circ}$ \\
\hline 2 vs. 3 & 1.22 & $0.83-1.79$ & 0.2991 & 0.80 & $0.53-1.23$ & 0.3031 \\
\hline 3 vs. 4 & 1.93 & $0.98-3.80$ & 0.0725 & 2.68 & $1.26-5.68$ & $0.0191^{\circ}$ \\
\hline 4 vs. 5 & 2.70 & $0.94-7.79$ & 0.0875 & 1.47 & $0.45-4.76$ & 0.5358 \\
\hline \multicolumn{7}{|l|}{ BCLC } \\
\hline 0 vs. A & 1.39 & $1.13-1.72$ & $0.0013^{\mathrm{a}}$ & 1.31 & $1.03-1.67$ & 0.0245 \\
\hline A vs. B & 1.65 & $1.31-2.05$ & $<0.0001^{\mathrm{a}}$ & 1.34 & $1.03-1.72$ & 0.0308 \\
\hline B vs. $\mathrm{C}$ & 0.94 & $0.63-1.38$ & 0.7710 & 1.23 & $0.77-1.91$ & 0.3712 \\
\hline
\end{tabular}

${ }^{\mathrm{a}} \mathrm{P}<0.05$. BCLC, Barcelona Clinic Liver Cancer; CLIP, Cancer of the Liver Italian Program; DFS, disease-free survival; OS, overall survival; JIS, Japan Integrated Staging score; MITS-CP, the mathematical integrated model for tumor staging with the Child-Pugh classification; MITS-LD, the mathematical integrated model for tumor staging with the degree of liver damage classification; MITS-ALBI, the mathematical integrated model for tumor staging with Albumin-Bilirubin grade; HR, hazard ratio. 
transforming both tumor number and size into a single parameter. The current study demonstrated that the NxS factor with 'user-friendly' cut-off values (i.e. 4 and 9) could be a simple and comprehensive measure of tumor burden to predict the prognosis of HCC patients following liver resection.

Notably, the NxS factor can be obtained from imaging modalities such as computed tomography (CT) or magnetic resonance imaging (MRI), and information on pathological vessel involvement is not needed. Although vascular invasion, one of the TNM staging components, is considered a prognostic factor, a peripheral vascular invasion is usually observed as microvascular invasion in the resected specimen and underestimated preoperatively $(29,30)$. Thus, unsuccessful preoperative assessment causes a potential limitation in the TNM and TNM-based systems due to the discrepancy between the pre- and postoperative status of HCC patients. In this regard, there is still room for the development of a novel tumor factor that is simple, robust, and does not need information on pathological vessel involvement. The $\mathrm{NxS}$ factor could solve these problems, and it was demonstrated that the patients' distribution of the NxS factor was relevant to that of the T factor of the TNM staging systems, especially UICC 8th, in the current study. Additionally, both tumor number and size of the largest tumor are also used as the main parameters of the TNM staging system $(22,31)$, and the current study confirmed that the MITS-LD model better predicted the prognosis of HCC patients than the TNM and TNM-based systems, such as the JIS score, M-JIS score, and ALBI-T score. Unlike the BCLC staging, the MITS models are not intended to be treatment allocation systems, however, the MITS models could be easy-to-use preoperative assessments for predicting HCC prognosis because the scores can be determined preoperatively via several imaging modalities and laboratory data.

Another goal of the MITS models is to develop a globally applicable staging classification. There is currently no globally accepted system for predicting HCC prognosis, and thus no common language on which to base treatment decisions and guide research. Our novel NxS factor is very simple and obtained anywhere and easily in daily practice. Thus, it may become one of the commonly used scores in many countries to predict prognosis of $\mathrm{HCC}$ patient. Although the prognosis of HCC patients is complex for various reasons, simple staging systems available anywhere are needed at first to compare differences the prognosis of HCC patients among the nations. Among the staging systems that combine tumor factor and liver function, the MITS-LD model, which integrates the degree of liver damage classification, provides the best prognostic ability. The degree of liver damage classification was proposed by the LCSGJ and incorporates the ICGR15 test instead of encephalopathy in the Child-Pugh classification system. The ICGR 15 test has been widely used in the field of surgery in Japan as a useful marker of hepatic function (32), but it is not routinely assessed in other parts of the world. Therefore, the MITS-LD model may not be validated in countries outside of Japan, but the MITS-ALBI model, which integrates the ALBI score (23) calculated using only serum albumin and total bilirubin, provided predictive accuracy for DFS. The ALBI score is a new model for assessing the severity of liver dysfunction and is a simpler, more objective, and evidence-based alternative to the Child-Pugh classification (21). Furthermore, Hiraoka et al (33) reported that the patients' distribution of the ALBI score was similar to that of the liver damage classification. Thus, the MITS-ALBI score can be a useful system for assessing HCC prognosis when and where the ICGR15 test cannot be obtained.

The present study had several limitations. First, the data on tumor characteristics in the current study were collected from postoperative histopathology reports. In fact, further analysis of preoperative and postoperative data collected prospectively is needed to investigate the prognostic ability of the MITS score in terms of its utility for preoperative assessment and a low discrepancy between the pre- and postoperative status of HCC patients. Second, the MITS models were developed and validated in Japan, and in the current study, $66.7 \%$ of $\mathrm{HCC}$ patients had evidence of $\mathrm{HCV}$ infection. This feature is different from Western countries, North America, Africa, and other Asian countries (34), and the present results require confirmation in different geographical areas. Third, the NxS factor only considers tumor morphology, and HCC patients with invasion of the major portal or hepatic vein branches were excluded in this study. Although macrovascular invasion has been well accepted as an established marker of a poor prognosis and is staged as an advanced group, such as BCLC stage C (14) or T4 of the TNM (UICC) staging system (31), the MITS models are not appropriate for HCC with macrovascular invasion. Fourth, this study investigated HCC patients who underwent complete surgical resection, and most patients were diagnosed at a relatively early stage with good liver function. In the current study, surgical resection was applied for $\mathrm{HCC}$ with $\leq 3$ lesions regardless of the tumor size in preoperative imaging modality, when preoperative liver function is Liver damage A or B. As expected, almost of all patients had HCC with $\leq 3$ lesions and there were only 20 patients $(2.1 \%)$ had multiple $\mathrm{HCC}$ with $\geq 4$ lesions in the resected specimen. Thus, validity of upper-intermediate and advanced HCC had been unknown. In this regard, further studies will be needed to evaluate whether the robustness of the MITS models in predicting HCC prognosis could be maintained in a cohort in which the majority of patients receive non-surgical treatment.

In patients with $\mathrm{HCC}$, the prediction of prognosis is complex compared with most solid tumors, because the prognosis and treatment of HCC depend on the tumor burden in addition to patient's underlying liver disease and liver functional reserve. It has been generally known that early recurrence of HCC, which is considered as residual intrahepatic metastasis, is the major risk factor affecting survival after hepatic resection. On the other hand, even when primary HCC is completely treated, recurrence is observed much more frequently in the form of multicentric carcinogenesis in the residual cirrhotic liver, because the potential for multicentric carcinogenesis increases with the progression of chronic liver disease and liver cirrhosis $(35,36)$. We have already demonstrated that the NxS factor is a powerful prognostic factor to predict early HCC recurrence (25), and in the current study, the MITS models had good stratification ability for DFS. Even now, there is no standard of care for adjuvant therapy for HCC patients who underwent hepatectomy. However, the prognostic score based on the data of patients who did not receive effective adjuvant therapy will be valuable and act as the standard when 
the effective adjuvant therapies to prevent early HCC recurrence are developed. Thus, we expect that the MITS models will help us better decide which patients need adjuvant therapy and which patients do not in the future. On the other hand, long-term OSs of MITS 0-2 were similarly poor prognosis, and these outcomes may derive from the underlying liver disease, liver functional reserve and multicentric carcinogenesis. In the future, effective antiviral agents such as direct acting antivirals for HCV (37) may prove an improvement long-term prognosis of $\mathrm{HCC}$ patients.

In conclusion, the $\mathrm{NxS}$ factor could be a comprehensive measure of tumor burden to predict the prognosis of HCC patients following liver resection, and the MITS-LD score provides better prognostic ability than known staging systems. The MITS-LD score could also be an easy-to-use preoperative assessment for predicting HCC prognosis, because the scores are very simple and can be determined preoperatively via several imaging modalities and laboratory data in daily practice.

\section{Acknowledgements}

The authors would like to thank Mr. Yuki Nakagami (Department of Translational Research and Developmental Therapeutics Against Cancer, Yamaguchi University School of Medicine) for his statistical assistance.

\section{Funding}

No funding was received.

\section{Availability of data and materials}

The datasets used and/or analyzed during the current study are available from the corresponding author on reasonable request.

\section{Authors' contributions}

YT designed the present study, analyzed and interpreted the patient data, and was a major contributor in writing the manuscript. YS, HM, SM and MN collected and interpreted the patient data. NS and ST interpreted the data and revised the manuscript. HW, SK and HE performed the surgical procedures, treated the specimens, and collected and interpreted the patient data. TU interpreted the data and revised the manuscript. HN performed the operations, interpreted the data and drafted the manuscript. All authors read and approved the final manuscript.

\section{Ethics approval and consent to participate}

The present study was approved by Institutional Review Board of Yamaguchi University Hospital [approval no. H27-141 (December 22, 2015)].

\section{Patient consent for publication}

Not applicable.

\section{Competing interests}

The authors declare that they have no competing interests.

\section{References}

1. Forner A, Llovet JM and Bruix J: Hepatocellular carcinoma. Lancet 379: 1245-1255, 2012.

2. Torre LA, Bray F, Siegel RL, Ferlay J, Lortet-Tieulent J and Jemal A: Global cancer statistics, 2012. CA Cancer J Clin 65: 87-108, 2015

3. Colvin H, Mizushima T, Eguchi H, Takiguchi S, Doki Y and Mori M: Gastroenterological surgery in Japan: The past, the present and the future. Ann Gastroenterol Surg 1: 5-10, 2017.

4. Kakeji Y, Takahashi A, Udagawa H, Unno M, Endo I, Kunisaki C, Taketomi A, Tangoku A, Masaki T, Marubashi S, et al: Surgical outcomes in gastroenterological surgery in Japan: Report of national clinical database 2011-2016. Ann Gastroenterol Surg 2: 37-54, 2017.

5. Henderson JM, Sherman M, Tavill A, Abecassis M, Chejfec G and Gramlich T: AHPBA/AJCC consensus conference on staging of hepatocellular carcinoma: consensus statement. HPB (Oxford) 5: 243-250, 2003.

6. Llovet JM, Ricci S, Mazzaferro V, Hilgard P, Gane E, Blanc JF, de Oliveira AC, Santoro A, Raoul JL, Forner A, et al: Sorafenib in advanced hepatocellular carcinoma. NEngl J Med 359: 378-390, 2008.

7. Kudo M, Finn RS, Qin S, Han KH, Ikeda K, Piscaglia F, Baron A Park JW, Han G, Jassem J, et al: Lenvatinib versus sorafenib in first-line treatment of patients with unresectable hepatocellular carcinoma: A randomised phase 3 non-inferiority trial. Lancet 391: 1163-1173, 2018.

8. Hazama S, Tamada K, Yamaguchi Y, Kawakami Y and Nagano H: Current status of immunotherapy against gastrointestinal cancers and its biomarkers: Perspective for precision immunotherapy. Ann Gastroenterol Surg 2: 289-303, 2018.

9. Kudo M, Chung H and Osaki Y: Prognostic staging system for hepatocellular carcinoma (CLIP score): Its value and limitations, and a proposal for a new staging system, the Japan integrated staging score (JIS score). J Gastroenterol 38: 207-215, 2003.

10. Nanashima A, Sumida Y, Morino S, Yamaguchi H, Tanaka K, Shibasaki S, Ide N, Sawai T, Yasutake T, Nakagoe T and Nagayasu T: The Japanese integrated staging score using liver damage grade for hepatocellular carcinoma in patients after hepatectomy. Eur J Surg Oncol 30: 765-770, 2004.

11. Harimoto N, Yoshizumi T, Sakata K, Nagatsu A, Motomura T, Itoh S, Harada N, Ikegami T, Uchiyama H, Soejima $\mathrm{Y}$ and Maehara Y: Prognostic significance of combined albumin-bilirubin and tumor-node-metastasis staging system in patients who underwent hepatic resection for hepatocellular carcinoma. Hepatol Res 47: 1289-1298, 2017.

12. Prospective validation of the CLIP score: A new prognostic system for patients with cirrhosis and hepatocellular carcinoma: The Cancer of the Liver Italian Program (CLIP) investigators. Hepatology 31: 840-845, 2000.

13. Tateishi R, Yoshida H, Shiina S, Imamura H, Hasegawa K, Teratani T, Obi S, Sato S, Koike Y, Fujishima T, et al: Proposal of a new prognostic model for hepatocellular carcinoma: An analysis of 403 patients. Gut 54: 419-425, 2005.

14. Llovet JM, Bru C and Bruix J: Prognosis of hepatocellular carcinoma: The BCLC staging classification. Semin Liver Dis 19: 329-338, 1999.

15. Arii S, Sata M, Sakamoto M, Shimada M, Kumada T, Shiina S, Yamashita T, Kokudo N, Tanaka M, Takayama T and Kudo M: Management of hepatocellular carcinoma: Report of consensus meeting in the 45th annual meeting of the Japan society of hepatology (2009). Hepatol Res 40: 667-685, 2010.

16. Yau T, Tang VY, Yao TJ, Fan ST, Lo CM and Poon RT: Development of hong kong liver cancer staging system with treatment stratification for patients with hepatocellular carcinoma. Gastroenterology 146: 1691-1700, 2014.

17. Pons F, Varela M and Llovet JM: Staging systems in hepatocellular carcinoma. HPB (Oxford) 7: 35-41, 2005.

18. Tokumitsu Y and Nagano H: Current HCC staging systems: Their uses and limitations. In: Hepatocellular Carcinoma: Diagnosis and treatment. Carr BI (ed). Springer, Basel, pp425-442, 2016.

19. Tokumitsu Y, Tamesa T, Matsukuma S, Hashimoto N, Maeda Y, Tokuhisa Y, Sakamoto K, Ueno T, Hazama S, Ogihara H, et al: An accurate prognostic staging system for hepatocellular carcinoma patients after curative hepatectomy. Int J Oncol 46: 944-952, 2015.

20. Kokudo N, Hasegawa K, Akahane M, Igaki H, Izumi N, Ichida T, Uemoto S, Kaneko S, Kawasaki S, Ku Y, et al: Evidence-based clinical practice guidelines for hepatocellular carcinoma: The Japan Society of Hepatology 2013 update (3rd JSH-HCC Guidelines). Hepatol Res 45: 12464, 2015. 
21. Pugh RN, Murray-Lyon IM, Dawson JL, Pietroni MC and Williams R: Transection of the oesophagus for bleeding oesophageal varices. Br J Surg 60: 646-649, 1973.

22. The general rules for the clinical and pathological study of primary liver cancer. Kanehara, Tokyo, pp 7-41, 2010.

23. Johnson PJ, Berhane S, Kagebayashi C, Satomura S, Teng M, Reeves HL, O'Beirne J, Fox R, Skowronska A, Palmer D, et al: Assessment of liver function in patients with hepatocellular carcinoma: A new evidence-based approach-the ALBI grade. J Clin Oncol 33: 550-558, 2015.

24. Mazzaferro V, Regalia E, Doci R, Andreola S, Pulvirenti A Bozzetti F, Montalto F, Ammatuna M, Morabito A and Gennari L: Liver transplantation for the treatment of small hepatocellular carcinomas in patients with cirrhosis. N Engl J Med 334: 693-699, 1996.

25. Tokumitsu Y, Sakamoto K, Tokuhisa Y, Matsui H, Matsukuma S, Maeda Y, Sakata K, Wada H, Eguchi H, Ogihara H, et al: A new prognostic model for hepatocellular carcinoma recurrence after curative hepatectomy. Oncol Lett 15: 4411-4422, 2018.

26. Mazzaferro V: Results of liver transplantation: With or without milan criteria? Liver Transpl 13 (11 Suppl 2): S44-S47, 2007.

27. Mazzaferro V, Llovet JM, Miceli R, Bhoori S, Schiavo M, Mariani L, Camerini T, Roayaie S, Schwartz ME, Grazi GL, et al: Predicting survival after liver transplantation in patients with hepatocellular carcinoma beyond the Milan criteria: A retrospective, exploratory analysis. Lancet Oncol 10: 35-43, 2009.

28. Hsu CY, Huang YH, Hsia CY, Su CW, Lin HC, Loong CC, Chiou YY, Chiang JH, Lee PC, Huo TI and Lee SD: A new prognostic model for hepatocellular carcinoma based on total tumor volume: The taipei integrated scoring system. J Hepatol 53: 108-117, 2010.

29. Kim BK, Han KH, Park YN, Park MS, Kim KS, Choi JS, Moon BS, Chon CY, Moon YM and Ahn SH: Prediction of microvascular invasion before curative resection of hepatocellular carcinoma. J Surg Oncol 97: 246-252, 2008.
30. Yamashita YI, Imai K, Yusa T, Nakao Y, Kitano Y, Nakagawa S, Okabe H, Chikamoto A, Ishiko T, Yoshizumi T, et al: Microvascular invasion of single small hepatocellular carcinoma $</=3 \mathrm{~cm}$ : Predictors and optimal treatments. Ann Gastroenterol Surg 2: 197-203, 2018.

31. Brierley JD, Gospodarowicz MK and Wittekind C (eds). TNM Classification of Malignant Tumours. 8th edition. Wiley-Blackwell, pp 80-82,2017.

32. Wakabayashi $\mathrm{H}$, Ishimura $\mathrm{K}$, Izuishi $\mathrm{K}$, Karasawa $\mathrm{Y}$ and Maeta H: Evaluation of liver function for hepatic resection for hepatocellular carcinoma in the liver with damaged parenchyma. J Surg Res 116: 248-252, 2004.

33. Hiraoka A, Kumada T, Michitaka K, Toyoda H, Tada T, Ueki H, Kaneto M, Aibiki T, Okudaira T, Kawakami T, et al: Usefulness of albumin-bilirubin grade for evaluation of prognosis of 2584 Japanese patients with hepatocellular carcinoma. J Gastroenterol Hepatol 31: 1031-1036, 2016.

34. Llovet JM, Burroughs A and Bruix J: Hepatocellular carcinoma. Lancet 362: 1907-1917, 2003.

35. Adachi E, Maeda T, Matsumata T, Shirabe K, Kinukawa N, Sugimachi K and Tsuneyoshi M: Risk factors for intrahepatic recurrence in human small hepatocellular carcinoma. Gastroenterology 108: 768-775, 1995.

36. Ikeda K, Saitoh S, Tsubota A, Arase Y, Chayama K, Kumada H, Watanabe $\mathrm{G}$ and Tsurumaru M: Risk factors for tumor recurrence and prognosis after curative resection of hepatocellular carcinoma. Cancer 71: 19-25, 1993.

37. Gao M, Nettles RE, Belema M, Snyder LB, Nguyen VN, Fridell RA, Serrano-Wu MH, Langley DR, Sun JH, O'Boyle DR II, et al: Chemical genetics strategy identifies an HCV NS5A inhibitor with a potent clinical effect. Nature 465: 96-100, 2010. 